Final version in Physical Review Letters 77, 3264 (1996)

\title{
Quantum Cryptography with Orthogonal States?
}

In a recent Letter [1], Goldenberg and Vaidman (GV) propose a new quantum cryptographical protocol "based on orthogonal states," and claim that it is essentially different from all existing quantum cryptosystems, which use non-orthogonal states as the carriers of information. This claim is unfounded: in the oldest protocol [2], known as BB84, the information sender (Alice) uses two orthogonal states, in a basis of her choice, for representing the values 0 and 1 of a bit. However, when a photon reaches the receiver (Bob), the latter does not know the basis used by Alice. He can in principle store that photon and wait until Alice publicly discloses her basis (in practice, it is easier to test the photon in a randomly chosen basis, and later to discard the corresponding bit, if it was tested with the wrong basis). An eavesdropper (Eve) has no such option of waiting, or discarding wrong choices. From her point of view, there are four possible states, not all orthogonal. She cannot acquire any information without a risk of perturbing these states. Only with the help of a time machine could eavesdropping be done with impunity.

The GV protocol has quite similar features. The process is represented in Fig. 1 as a spacetime diagram, for more clarity. This figure differs from the one in ref. [1], where the storage rings $\mathrm{SR}_{1}$ and $\mathrm{SR}_{2}$ were drawn very small, while actually they are longer than the distance from Alice to Bob. Here, to avoid such a scale distortion, I represented the storage rings by zigzag lines (as if, instead of coiled optical fibers, there were mirrors between which the photons bounced many times). Alice uses two different input ports (two orthogonal states) in order to materialize bit values 0 and 1, and Bob likewise detects them as othogonal states. On the other hand, the states accessible to Eve are not orthogonal, just as in the BB84 protocol. With the standard notations of quantum optics, the quantum state between the two beamsplitters is $\left|\psi_{ \pm}\right\rangle=2^{-1 / 2}(|0\rangle|1\rangle \pm|1\rangle|0\rangle)$, where the first and second kets refer to the two branches of the interferometer, and the \pm sign corresponds to bit values 0 and 1 , respectively. These two orthogonal states may also be represented by density matrices, $\rho_{ \pm}=\left|\psi_{ \pm}\right\rangle\left\langle\psi_{ \pm}\right|$. 
As seen in the figure, Eve can access the information carrier only in two time windows, and in each one of them she controls just one branch of the interferometer. If Eve is restricted to performing only instantaneous measurements, the other branch of the interferometer is not accessible to her. The results of an instantaneous measurement in a single branch are then obtained by "tracing out" the other branch in $\rho_{ \pm}$. This gives a reduced density matrix, $\rho_{ \pm}^{\prime}=\frac{1}{2}(|0\rangle\langle 0|+| 1\rangle\langle 1|)$, which is the same for both branches, and also the same for bit values 0 and 1 . The $\rho_{ \pm}^{\prime}$ states, as seen by Eve, are not orthogonal. They are identical. The meaning of $\rho_{ \pm}^{\prime}$ is that Eve has a $50 \%$ chance of getting the vacuum, or getting one particle.

If, on the other hand, Eve is not restricted to instantaneous measurements, she can use an apparatus similar to Bob's, with a delay line, and then she can identify the states $\left|\psi_{ \pm}\right\rangle$ with certainty. However, the resulting delay is observable by Bob, exactly as it would be in the BB84 protocol if Eve stored the message carriers until after Alice announced the basis she used. Moreover, as shown by GV, it is essential that Alice announces the exact time of emission of each photon only after the latter was received by Bob. Otherwise, Eve could successfully eavesdrop by introducing a dummy particle.

In summary, the GV protocol has many features similar to those of BB84. From the point of view of Alice, each bit has its value represented by one of two orthogonal states, but it must be accompanied by delayed information: namely, the basis chosen by Alice, or the exact time of emission (the delayed information may be either classical, or encoded into orthogonal quantum states of additional photons).

Moreover, it is possible to imagine other protocols, interpolating between those of GV and BB84. Their common feature is that information is sent in two consecutive steps, and security is achieved by withholding the second piece of information until after Bob receives the first one, and Eve can no longer access it. For example, Alice could publicly declare when she sends a photon, and, instead of using two different bases as in BB84, she would use two identical GV interferometers, and disclose her choice only at a later time. A general theory of eavesdropping on two-step quantum protocols is an interesting problem, worth being investigated in more detail.

GV also propose a "relativistic" version of their protocol, with widely separated paths 
instead of a time delay. This method is not secure: a team of eavesdroppers could use mirrors to redirect the photon paths toward a common inspection center, and thence to Bob, without arousing suspicion.

I thank Lior Goldenberg and Lev Vaidman for an advance copy of their Letter. This work was supported by the Gerard Swope Fund, and the Fund for Encouragement of Research.

Asher Peres

Department of Physics

Technion-Israel Institute of Technology

32000 Haifa, Israel

PACS numbers: 03.65.Bz, 89.70.+c

1. L. Goldenberg and L. Vaidman, Phys. Rev. Lett. 75, 1239 (1995).

2. C. H. Bennett and G. Brassard, in Proc. IEEE International Conf. on Computers, Systems and Signal Processing, Bangalore, India (IEEE, New York, 1984) p. 175.

\section{Caption of figure}

FIG. 1. The photon carrying the information is split into two wave packets, moving with velocity $c$. Eve, restricted to $x \simeq 0$, can test at most one branch of its wave function at any time (in one of the shaded areas). 


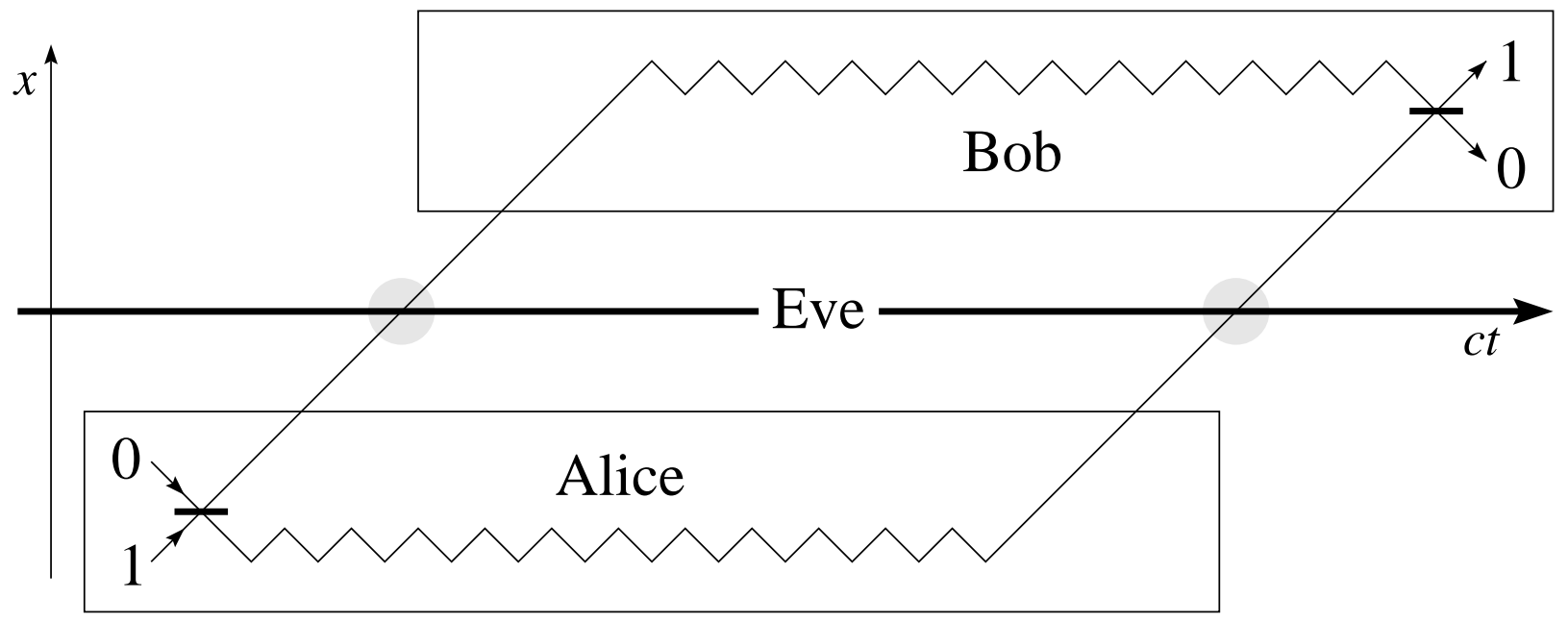

Peres - Quantum cryptography with orthogonal states? 